\title{
The Transcription Factors GATA-1 and GATA-4 Have Opposite Effects on DNA Expression Driven by an Amh Promoter
}

\author{
David W. Dresser \\ 3IR, The Ashworth Laboratory, The University of Edinburgh, Edinburgh, UK \\ Email: david@daviddresser.co.uk
}

Received 12 May 2014; revised 11 June 2014; accepted 10 July 2014

Copyright (C) 2014 by author and Scientific Research Publishing Inc. This work is licensed under the Creative Commons Attribution International License (CC BY). http://creativecommons.org/licenses/by/4.0/ cc) (7) Open Access

\begin{abstract}
An Amh promoter driving expression of a reporter gene (d2EGFP) has been used to analyze the role of two specific promoter transcription factor binding elements. In addition a downstream (3') enhancer (DE) was also investigated. The transcription factors GATA-1 and GATA-4 had opposite effects, the former being incremental and the latter decremental. The quantitative balance between these two factors may provide a degree of control over the level of gene expression.
\end{abstract}

\section{Keywords}

SMAT-1, Mouse Pre-Pubertal Sertoli, In Vitro, Amh Promoter, d2EGFP, Transcription Factors, GATA-1, GATA-4

\section{Introduction}

Study of the role of Sertoli cells in sexual differentiation and in particular the role of the protein AMH [1], can be facilitated using appropriate cell lines in vitro. Such a cell is SMAT-1 [2] a mouse prepubertal Sertoli cell line, adherent to tissue culture plasticware and therefore convenient for experiments involving transfection with DNA plasmid constructs. Here it is reported that a green fluorescent reporter gene (d2EGFP), driven by a promoter such as that of Amh (Figure 1), makes it feasible to measure the rate of gene expression at the level of individual cells by flow cytometry. Previously it was shown that mutation of some putative transcription factor binding elements in the promoter of Amh led to a significant lowering of reporter gene expression [3] [4]: however mutation of one particular element (proximal Gata) resulted in an increase in expression. Furthermore it was demonstrated that a 3' (downstream) region acted as an enhancer (DE) when bridged to the Wilms tumour factor-1 element situated between the tata box and the start of translation of the Amh promoter [5]. In the current 


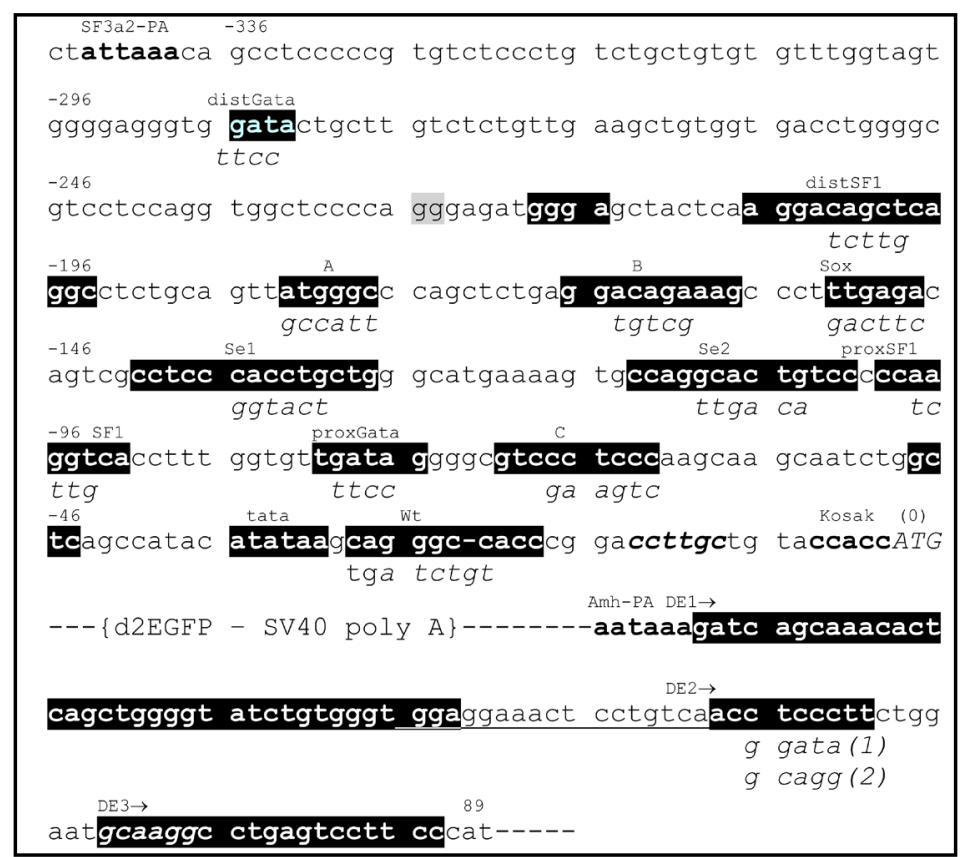

Figure 1. Nucleotide sequence (5' to 3') of a mouse Amh promoter. SF3a2-PA is the polyadenylation signal of an upstream gene coding for a spliceosome component [3]. Potential promoter elements (white on black) are identified on the basis of sequence similarity with human, rat and other mammalian Amh promoter sequences: the order of elements is conserved. These potential elements are highlighted white on black and identified by superscript titles, with mutated sequences indicated as subscripts. Where possible the superscript titles are defined by their affinity for known transcription factors. The start of translation (0) is position 8647 in GenBank mouse genomic nucleotide sequence X83733. DE is a downstream enhancer starting at the polyadenylation signal for Amh: this element was inserted in the d2EGFP vector at a MluI site as indicated in this figure. The MluI site replaces an AflII site which was in the vector as supplied by Invitrogen. Previously it was shown that mutation of distSF1; sox; Se1; and proxSF1; resulted in a significant reduction in EGFP expression. In contrast mutation of proxGata resulted in a small but significant increase in expression.

paper potential competition between two members of the GATA family of transcription factors, for a limited number of recogniseable Gata elements in the Amh promoter, has been investigated as a possible mechanism for fine-tuning gene expression.

\section{Materials \& Methods}

SMAT-1 cells [2] were grown adherently to tissue culture plasticware in DMEM-F12, 10\% foetal calf serum plus penicillin and streptomycin: except that for transfection using LipofectAmine 2000 (Invitrogen) the antibiotic was omitted. For the transfection procedure cultures of $\sim 10^{5}$ cells per well in Costar-24 plates were incubated for 24 hrs to allow cell adhesion to be completed. The cells were then transfected using $800 \mathrm{ng}$ of EGFP plasmid vector DNA together with $2 \mu \mathrm{l}$ LipofectAmine per well. After a further 42 - 48 hrs culture at $37^{\circ}$ in air with $5 \% \mathrm{CO}_{2}$, the cells were harvested for analysis in a flow cytometer.

The flowcytometry procedure which has been described in detail [4] was set up to exclude all autofluorescent cells, to register all live cells emitting green fluorescence and in addition, the ability to assay cells emitting red fluorescence when ERFP was used with or instead of EGFP. Results from quadruplicate cultures for each group were recorded, analysed and illustrated using Graph Pad Prism.

Site directed mutagenesis (SDM), by double overlapping extension PCR [6] [7] was used to change the nucleotide sequence of promoter and enhancer elements, as described previously [3]-[5].

Plasmid expression vectors for GATA-1 [8], GATA-4 [9] and plasmids producing third-party (control) DNA, were grown in DH10B (E. coli) bacteria in LB medium with ampicillin. All plasmid DNAs were prepared using 
Qiagen mini-maxi kits.

\section{Results}

Figure 1 is a map of the mouse Amh promoter region and associated putative enhancer elements which are highlighted as white on black with mutated versions of the elements indicated as subscript sequences. Previously published experimental data [3]-[5] showed that mutation of elements; distal SF1; Sox; Se1; and proximal SF1 play a positive role in promotion of reporter gene expression. Whereas mutation of the proximal Gata element showed that this element probably played a negative role.

To explore the role of recognizable Gata elements in the Amh promoter region, plasmid DNAs of GATA-1 and GATA-4 transcription factor expression vectors were compared, together with third-party control DNAs. Figure 2 illustrates the result of one of several experiments in which plasmid DNA of GATA-1; GATA-4; and a control were added to the transfection mixture. Two other control plasmid DNAs gave similar results to those seen in Figure 2. Seven experiments, normalized on the basis of their responses to the Amh promoter alone, are summarised in Figure 3. This normalization is necessary because of day-to-day differences in culture conditions and differences between flow cytometer machines.

GATA-1 plasmid added to Amh promoter DNA in the transfection mixture resulted in a $>$ four-fold increase in expression, whilst addition of GATA-1 to an Amh promoter linked to a downstream enhancer (DE) leads to $<$ two-fold increase (Figure 4 and Figure 5). This suggests that there is a natural maximum rate of expression beyond which the system cannot be pushed.

In the next set of experiments, GATA-1 or GATA-4 plasmid DNAs were added to Amh promoters in which distal Gata; proximal Gata or both Gata elements were mutated. The results are shown in Figure 6 and Figure 7. When both Gata elements were mutated (group D), the addition of GATA-1 to the transfection mixture, resulted in a decreased expression, contrasting with the increased expression seen when neither of the elements, or only

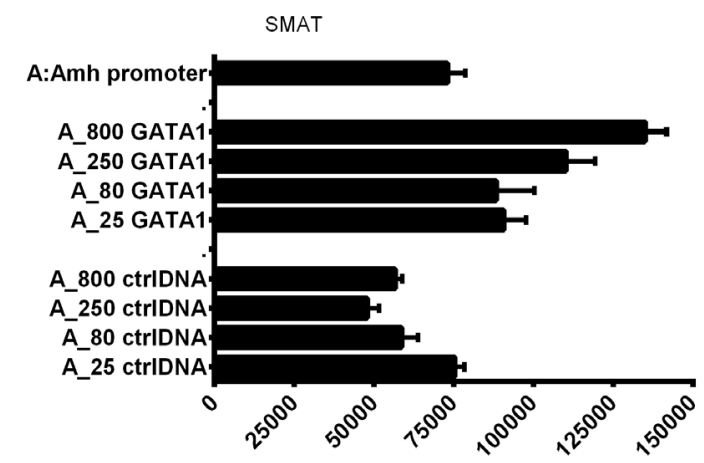

(a)

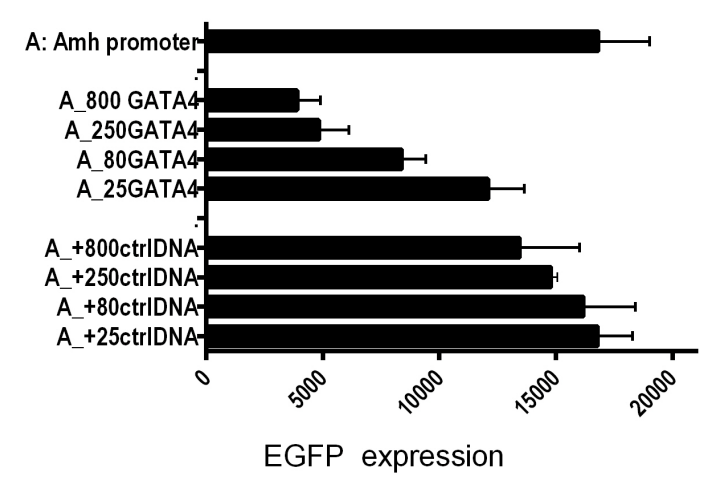

(b)

Figure 2. The addition of GATA-1; GATA-4 and control plasmid DNAs to the transfection mixture consisting of LipofectAmine 2000 with 800 ng EGFP plasmid DNA per culture in a Costar-24 plate. UPPER addition of GATA-1 and control (ctrl) DNA. LOWER addition of GATA-4 and ctrl DNA. The two GATAs have diametrically opposite dose related effects. 


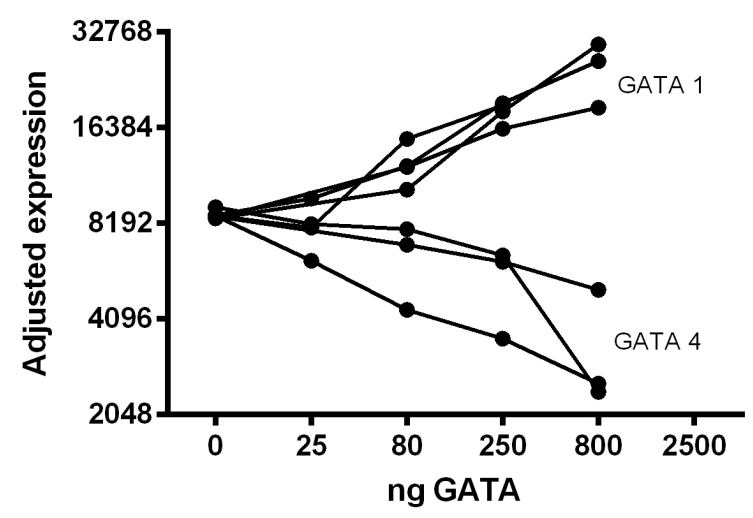

Figure 3. A summary of 7 experiments similar to those illustrated in Figure 2. The values are normalized on the basis of the respective responses to the Amh promoter alone. The differing effects of added GATA-1 and GATA-4 are manifest. Here and in subsequent figures plasmid DNA names are capitalized (viz. GATA-1) and promoter elements are partially in lower case (viz. distGata).

\section{Amh promoter}

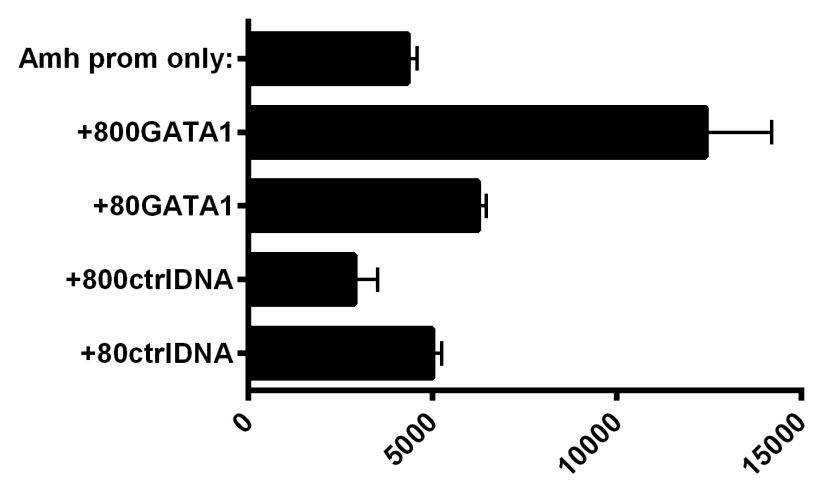

(a)

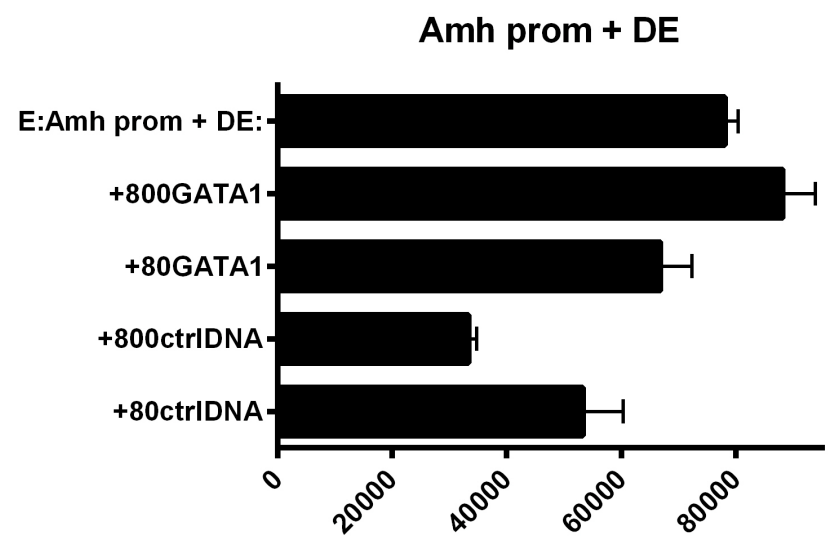

EGFP expression

(b)

Figure 4. A comparison of d2EGFP expression driven by Amh promoters with and without the 3' enhancer DE. The effect of the addition of GATA-1 to the transfection mixture is illustrated. The results in the UPPER and LOWER graphs were obtained on the same day using the same batch of SMAT cells: the expression values are therefore comparable. The relative increment due to the addition of GATA-1 is much greater than that obtained with the promoter without the DE enhancer. 


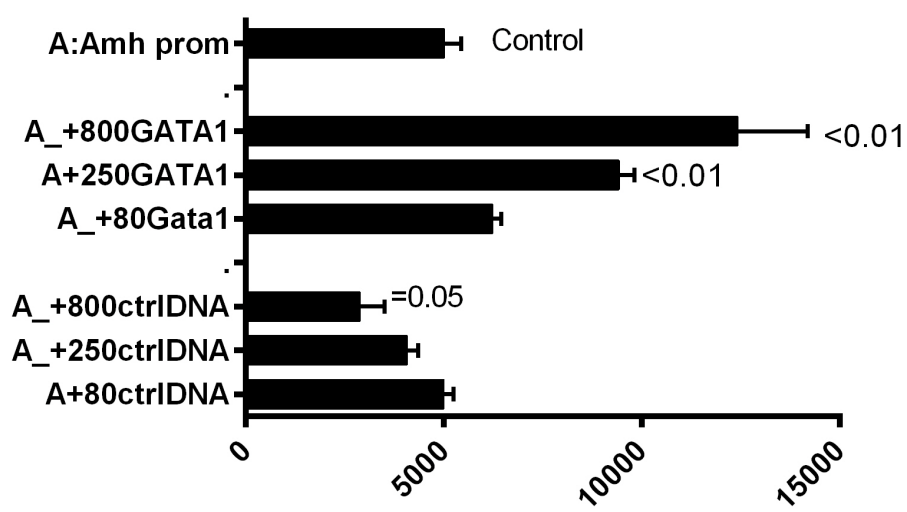

(a)

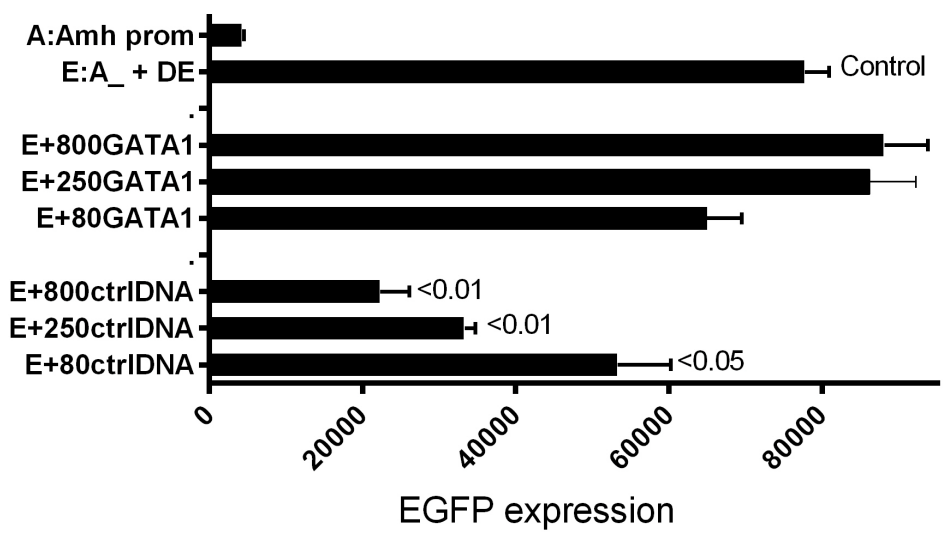

(b)

Figure 5. A further comparison of reporter gene expression consequent to the addition of GATA-1 DNA to the EGFP reporter gene-Amh promoter complex, with and without the DE enhancer.

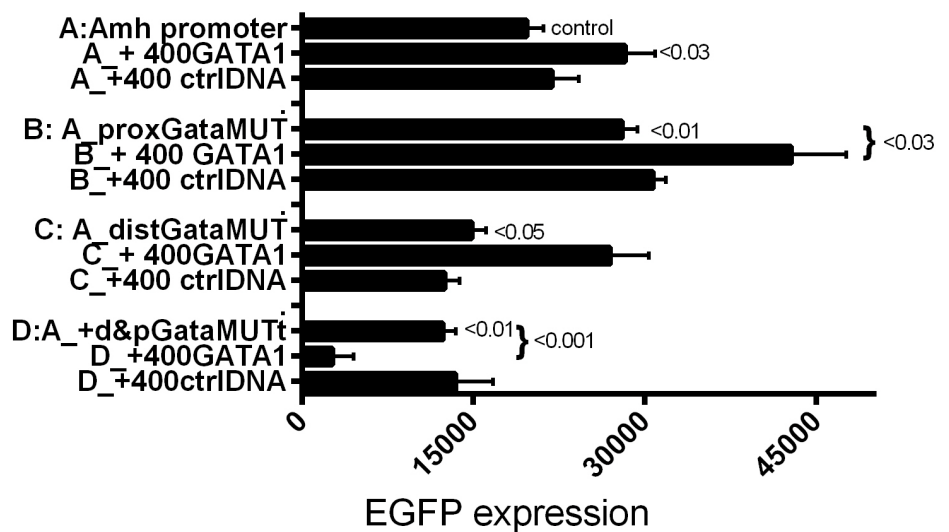

Figure 6. SMAT-1 cells transfected with d2EGFP driven by Amh promoters with and without mutated Gata elements; group B proximal Gata; C distal Gata; and D both Gatas mutated. The effect on expression with and without GATA-1 added to the transfection mixture. In a second experiment a similar result was obtained (data not included).

the proximal Gata, was mutated. Data illustrated in Figure 7 show a preference of individual GATAs for particular Gata elements. For example, changes in EGFP expression driven by Amh promoters with proximal (B); distal (C); or both Gata elements mutated (D) added to the transfection mixture, to which was also added GATA-1 or GATA-4. When the proximal Gata element was mutated additional GATA-1 had no effect and 
GATA-4 reduced expression: implying that GATA-4 may act preferentially through the distal Gata element. The converse situation is logically comparable: this is where a mutated distal Gata element was combined wirh added GATA-1, resulting in an increased expression and added GATA-4 had no effect. In contrast mutation of both Gata elements resulted in GATA-4 having no effect and GATA-1 a suppressive effect on EGFP expression (Figure 6 and Figure 7) this last point was a surprise so a group D experiment was repeated, using the same clone of double mutated Amh promoter DNA, which provided the same result. A convincing explanation for this observation of a GATA-1 suppressive effect is not yet available.

In the next set of experiments GATA-1 and GATA-4 plasmid DNAs were added to Amh promoters in the transfection mixture, in different proportions. These results are illustrated in Figure 8 and Figure 9, where it can be seen that a 50:50 mixture seems to result in the "GATA-4 effect" predominating.

Results obtained with control (ctrl) DNA, illustrated in Figures 4-9, are compatible with there being passive competition for "space" in the LipofectAmine vehicle used for transfection. In addition there may be competition at a functional GATA-1 v. GATA-4 level. Therefore it is not surprising that a mjxture of GATAs and ctrlDNAs leads to the equivocal results seen in Figure 9, where GATA and ctrl plasmids were mixed at the time of transfection.

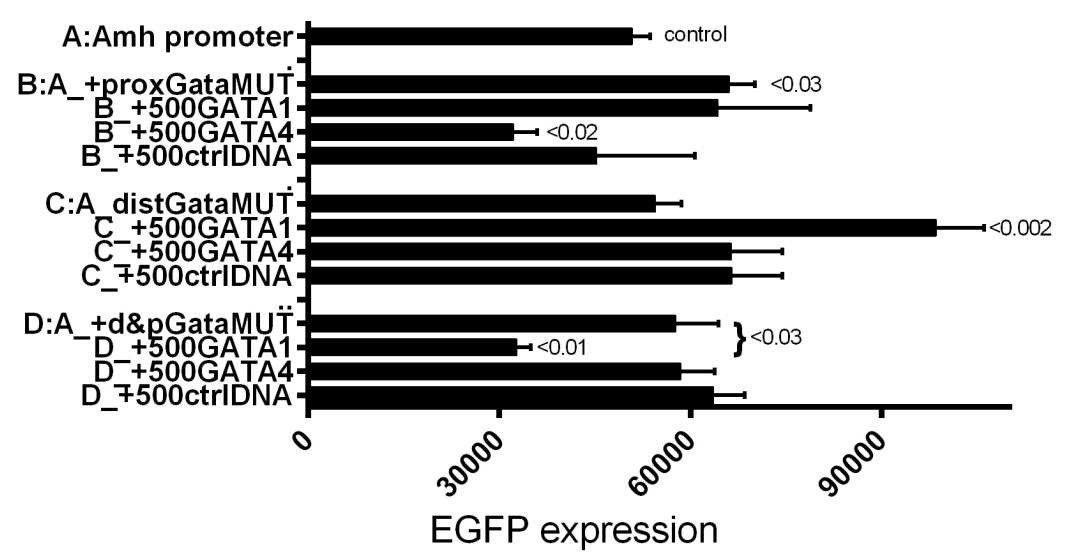

Figure 7. Similar to Figure 6 but including groups with GATA-4 as well as GATA-1. With a proximal Gata mutated, addition of GATA-4 decreases expression but added GATA-1 has no effect. When the distal Gata element is mutated (C) the opposite result was obtained. This suggests that GATA-4 may have at least a mild preference for the distal Gata site and GATA-1 a preference for the proximal Gata site. This provisional conclusion depends on there not being an other GATA-binding element.

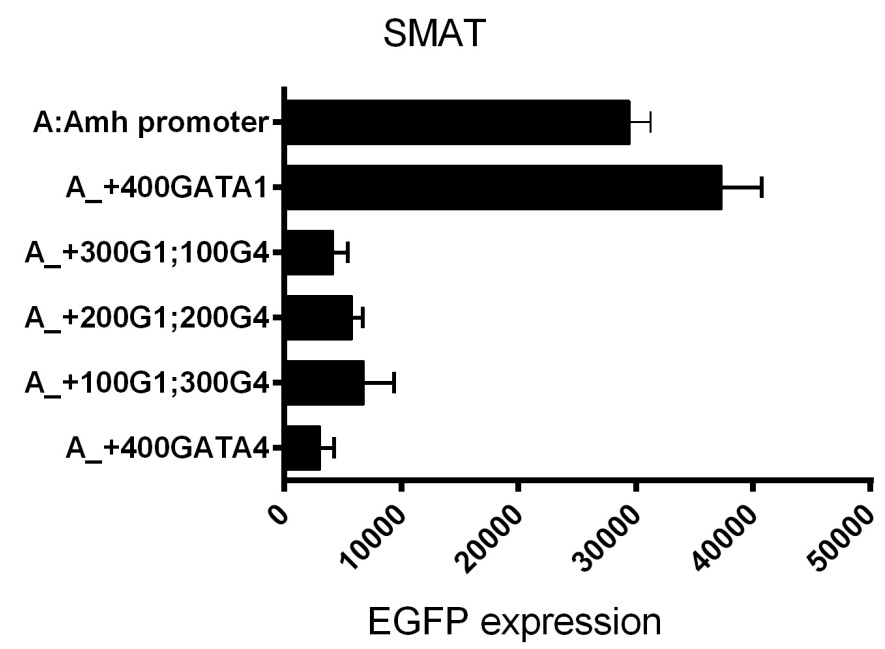

Figure 8. The effect of adding different proportions of GATA-1 and GATA-4 to the transfection mixture. Using a total of $400 \mathrm{ng}$ of added GATA plasmid DNA per culture. 


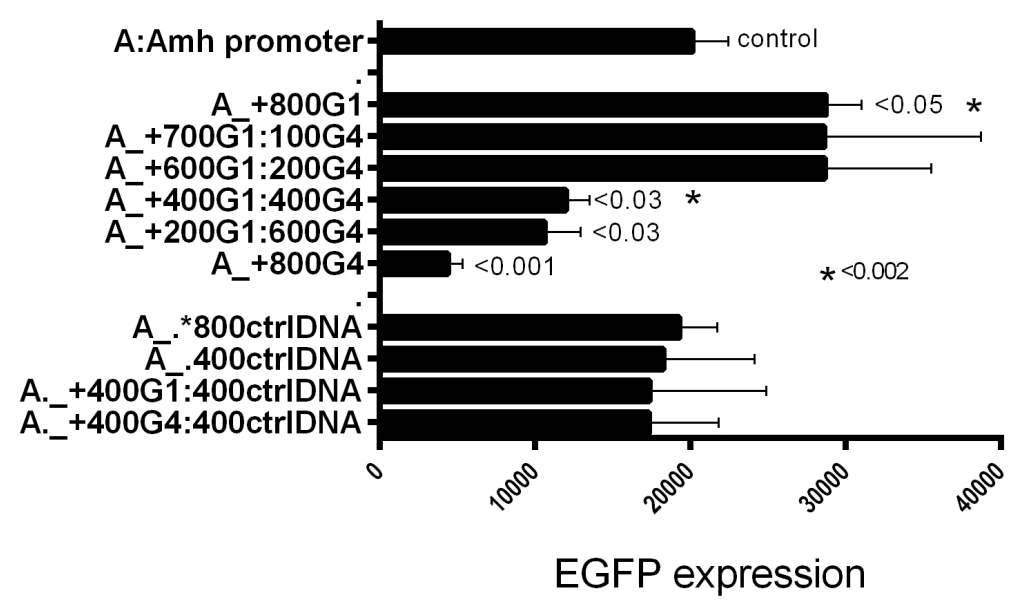

Figure 9. Similar to Figure 8 but with a total of 800 ng GATA plasmid DNA: at this level, 50\% GATA-4 seems to override the incremental effect of GATA1. It was assumed that the two GATA plasmid vectors expressed at similar rates.

\section{Discussion}

In vitro analysis of gene expression in appropriate cell lines has both advantages and disadvantages over in vivo studies. For example SMAT-1 a mouse pre-pubertal Sertoli cell line [2], can be grown in replicate cultures to allow many different experimental variables to be evaluated in a wide ranging and economical manner. However it must be acknowledged that many extra-cellular factors, which may be present in the intact animal, are likely to be missing in a cell culture vessel. Bearing this caveat in mind, it seemed sensible to proceed with the in vitro approach which can point the way to the selection of a limited number of critical in vivo approaches.

Amh promoters with various putative transcription factor binding elements mutated (Figure 1), were used to drive the expression of a green fluorescent reporter gene (d2EGFP) [3]-[5]. In some experiments a downstream enhancer (DE) was inserted at a 3' site in the EGFP DNA plasmid, at a position equivalent to that in the unmodified genomic Amh sequence (Figure 1). The role of two potential transcription factors, GATA-1 and GATA-4, was studied by adding DNA plasmid expression vectors to the DNA mixture used to transfect the cultured SMAT cells. That the addition of GATA-1 increases expression and GATA-4 has an opposite effect, is illustrated in Figure 4, which summarises the result of seven experiments performed over a period of several months. While the qualitative difference between GATA- 1 and GATA- 4 is unequivocal, the minutae of quantitative differences are less precise.

Presence of the downstream enhancer (DE) boosts expression of the reporter gene by a considerable margin over the level of expression seen in constructs of the Amh promoter without DE [5]. Similarly GATA-1 added to an Amh promoter lacking DE results in a significant increase in the level of expression. However when DE and GATA-1 are combined, there is a significantly less than additive increase in level of expression (Figure 4 and Figure 5). This suggests that each component on its own can push expression close to the maximum physiological level possible in this in vitro system.

A gene involved in development is likely to be part of an (epigenetic) interactive cascade of factors. The approach described in this paper may help to sketch out a snap-shot at one time-point but be unable to throw much light on the expression of upstream genes for individual transcription factors (tf) or even further upstream the tf of the tf.

A source of confusion, in the in vitro transfection experiments described here may be the presence in individual pre-pubertal SMAT-1 cells of a complete set of genomic genes related to Amh expression. However the presence of the products of "upstream" genes may be a key to determining the pre-pubertal status of SMAT cells and the status of post-pubertal cells and their failure to support Amh promoter driven expression of EGFP [5].

Data from a large body of published sources, largely based on in vivo experimentation [10]-[16], show that where similar points in development are compared there is a similarity between the two approaches. For example in vivo experiments by Arango et al. [12] showed that mutation of the (proximal) SF1 and Sox elements in the promoter of Amh led to an impairment of AMH expression. Similarly de Santa Barbara et al. [13] showed 
that the elements which controlled expression by mouse cells ex-vivo also played a key role in human testicular development. Also, Miamota et al. [14] seemed to demonstrate a functional relationship between GATA-4 and the Wilms tumour-1 binding protein. The observation that added GATA-1 in the transfection mixture led to an increased expression of the reporter gene, conflicts with the conclusion of Beau et al. [15] that GATA-1 acting at puberty, has a repressor function in Amh expression. However their conclusion may be compatible with the increased response seen when the proximal Gata element is mutated: the anomaly revealed here requires further investigation. Other somewhat more oblique studies throw some light on the role of GATA tf proteins on the expression of genes involved in sexual differentiation [16]-[23].

\section{Acknowledgements}

I thank Dr. N. Di Clemente for providing the SMAT-1 cells; the University of Edinburgh for an Emeritus Fellowship; Professor M. Yamamoto, Center for TARA Sukuba, for GATA-1; Addgene for GATA-4; Professor Rick Maizels and his colleagues for the hospitality of the lab; and Dr. R. J. M. (Iain) Wilson for a critical reading of a draft of this paper.

\section{References}

[1] Josso, N., Picard, J-Y., Rey, R. and di Clemente, N. (2006) Testicular anti-Mullerian Hormone: History, Genetics, Regulation and Clinical Applications. Pediatric Endocrinology Reviews, 3, 347-358.

[2] Belville, C, Jamin, S.P., Picard, J-Y., Josso, N. and di Clemente, N. (2005) Role of Type I Receptors for Anti-Mullerian Hormone in the SMAT-1 Sertoli Cell Line. Oncogene, 24, 4984-4992. http://dx.doi.org/10.1038/sj.onc.1208686

[3] Dresser, D.W. and Guerrier, D. (2005) Candidate Sertoli Cell Specific Promoter Element for a TGFbeta Family Member (Amh) and a 3' UTR Enhancer/Repressor for the Same Gene. Gene, 363, 159-165.

http://dx.doi.org/10.1016/j.gene.2005.08.004

[4] Dresser, D.W. (2012) Mutated Elements of a Complex Promoter (Amh) Can Help to Demonstrate the Role of Certain Elements in Controlling Differential Gene Expression. American Journal of Molecular Biology, 2, 351-358. http://dx.doi.org/10.4236/ajmb.2012.24036

[5] Dresser, D.W. (2013) Interaction between the Wilms Tumour Factor-1 Element in the Promoter of Amh and a Downstream Enhancer Is Required for a Strong Expression of the Gene in Pre-Pubertal Sertoli Cells. American Journal of Molecular Biology, 3, 165-172. http://dx.doi.org/10.4236/ajmb.2013.33022

[6] Vandevar, M.A., Weiner, M.P., Hutton, C.J. and Batt, C.A. (1988) A Simple and Rapid Method for the Selection of Oligodeoxynucleotide-Directed Mutants. Gene, 65, 129-133. http://dx.doi.org/10.1016/0378-1119(88)90425-8

[7] Ho, S.N., Hunt, H.D., Horton, R.M., Pullen, J.K. and Pease, L.R. (1989) Site-Directed Mutagenesis by Overlap Extension Using the Polymerase Chain Reaction. Gene, 77, 51-59. http://dx.doi.org/10.1016/0378-1119(89)90358-2

[8] Wakabayasht, J., Yomonida, K., Nakajuma, O., et al. (2003) GATA-1 Testis Activation Region Is Essential dor Sertoli Cell-Specific Expression of GATA-1 Gene in Transgenic Mouse. Genes to Cells, 8, 619-630. http://dx.doi.org/10.1046/j.1365-2443.2003.00658.x

[9] Arceci, R.J., King, A.A., Simon, M.C., et al. (1993) Mouse GATA-4: A Retinoic Acid-Inducible GATA-Binding Transcription Factor Expressed in Endodermally Derived Tissues and Heart. Molecular and Cellular Biology, 13, 2235-2246.

[10] Munsterberg, A. and Lovell-Badge, R. (1991) Expression of the Mouse Anti-Mullerian Hormone Gene Suggests a Role in Both Male and Female Sexual Differentiation. Development, 113, 613-624.

[11] Takada, S. and Koopman, P. (2003) Origin and Possible Roles of the Sox8 Transcription Factor Gene during Sexual Development. Cytogenetic and Genome Research, 101, 212-218. http://dx.doi.org/10.1159/000074339

[12] Arango, N.A., Lovell-Badge, R. and Behringer, R.R. (1999) Targeted Mutagenesis of the Endogenous Mouse Mis Gene Promoter: In Vivo Definition of Genetic Pathways of Vertebrate Sexual Development.Cell, 99, 409-419. http://dx.doi.org/10.1016/S0092-8674(00)81527-5

[13] De Santa Barbara, P., Moniot, B., Poulat, F. and Berta, P. (2000) Expression and Subcellular Localization of SF-1, SOX9, WT1, and AMH Proteins during Early Human Testicular Development. Developmental Dynamics, 217, 293298. http://dx.doi.org/10.1002/(SICI)1097-0177(200003)217:3<293::AID-DVDY7>3.0.CO;2-P

[14] Miamota, Y., Taniguchi, H., Hamel, F., Silversides, D.W. and Viger, R. (2008) A GATA4/WT1 Cooperation Regulates Transcription of Genes Required for Mammalian Sex Determination and Differentiation. BMC Molecular Biology, 9, 44-62. http://dx.doi.org/10.1186/1471-2199-9-44 
[15] Beau, C., Rauch, M., Joulin, V., Jegou, B. and Guerrier, D. (2000) GATA-1 Is a Potential Repressor of Anti-Müllerian Hormone Expression during the Establishment of Puberty in the Mouse. Molecular Reproduction and Development, 56, 124-138. http://dx.doi.org/10.1002/(SICI)1098-2795(200006)56:2<124::AID-MRD2>3.0.CO;2-J

[16] Schepers, G., Wilson, M., Wilhelm, D. and Koopman, P. (2003) SOX8 Is Expressed during Testis Differentiation in Mice and Synergizes with SF1 to Activate the Amh Promoter in Vitro. The Journal of Biological Chemistry, 278, 28101-28108. http://dx.doi.org/10.1074/jbc.M304067200

[17] Lovell-Badge, R., Canning, A. and Sekido, R. (2002) Sex-Determining Genes in Mice: Building Pathways. Novartis Foundation Symposia, 244, 4-18. http://dx.doi.org/10.1002/0470868732.ch2

[18] Furuhata, A., Murakami, M., Ito, H., et al. (2009) GATA-1 and GATA-2 Binding to 3' Enhancer of WT1 Gene Is Essential for Its Transcription in Acute Leukemia and Solid Tumor Cells. Leukemia, 23, 1270-1277. http://dx.doi.org/10.1038/leu.2009.13

[19] Mazaud Guittot, S., Tetu, A., Legault, E., Pilon, N., Silversides, D.W. and Viger, R.S. (2007) The Proximal Gata4 Promoter Directs Reporter Gene Expression to Sertoli Cells during Mouse Gonadal Development. Biology of Reproduction, 76, 85-95. http://dx.doi.org/10.1095/biolreprod.106.055137

[20] Oreal, E., Mazaud, S., Picard, J-Y., Magre, S. and Carre-Eusebe, D. (2002) Different Patterns of Anti-Mullerian Hormone Expression, as Related to DMRT1, SF-1, WT1, GATA-4, Wnt-4, and Lhx9 Expression, in the Chick Differentiating Gonads. Developmental Dynamics, 225, 221-232. http://dx.doi.org/10.1002/dvdy.10153

[21] Viger, R.S., Taniguchi, H., Robert, N.M. and Tremblay, J.J. (2004) Role of the GATA Family of Transcription Factors in Andrology. Journal of Andrology, 25, 441-452.

[22] Viger, R.S., Silversides, D.W. and Tremblay, J.J. (2004) New Insights into the Regulation of Mammalian Sex Derermination and Male Sex Differentiation. Vitamins Hormones, 70, 387-413. http://dx.doi.org/10.1016/S0083-6729(05)70013-3

[23] Sinclair, A., Smith, C., Western, P. and McClive, P. (2002) A Comparative Analysis of Vertebrate Sex Determination. Novartis Foundation Symposia, 244, 102-114. http://dx.doi.org/10.1002/0470868732.ch10 
Scientific Research Publishing (SCIRP) is one of the largest Open Access journal publishers. It is currently publishing more than 200 open access, online, peer-reviewed journals covering a wide range of academic disciplines. SCIRP serves the worldwide academic communities and contributes to the progress and application of science with its publication.

Other selected journals from SCIRP are listed as below. Submit your manuscript to us via either submit@scirp.org or Online Submission Portal.
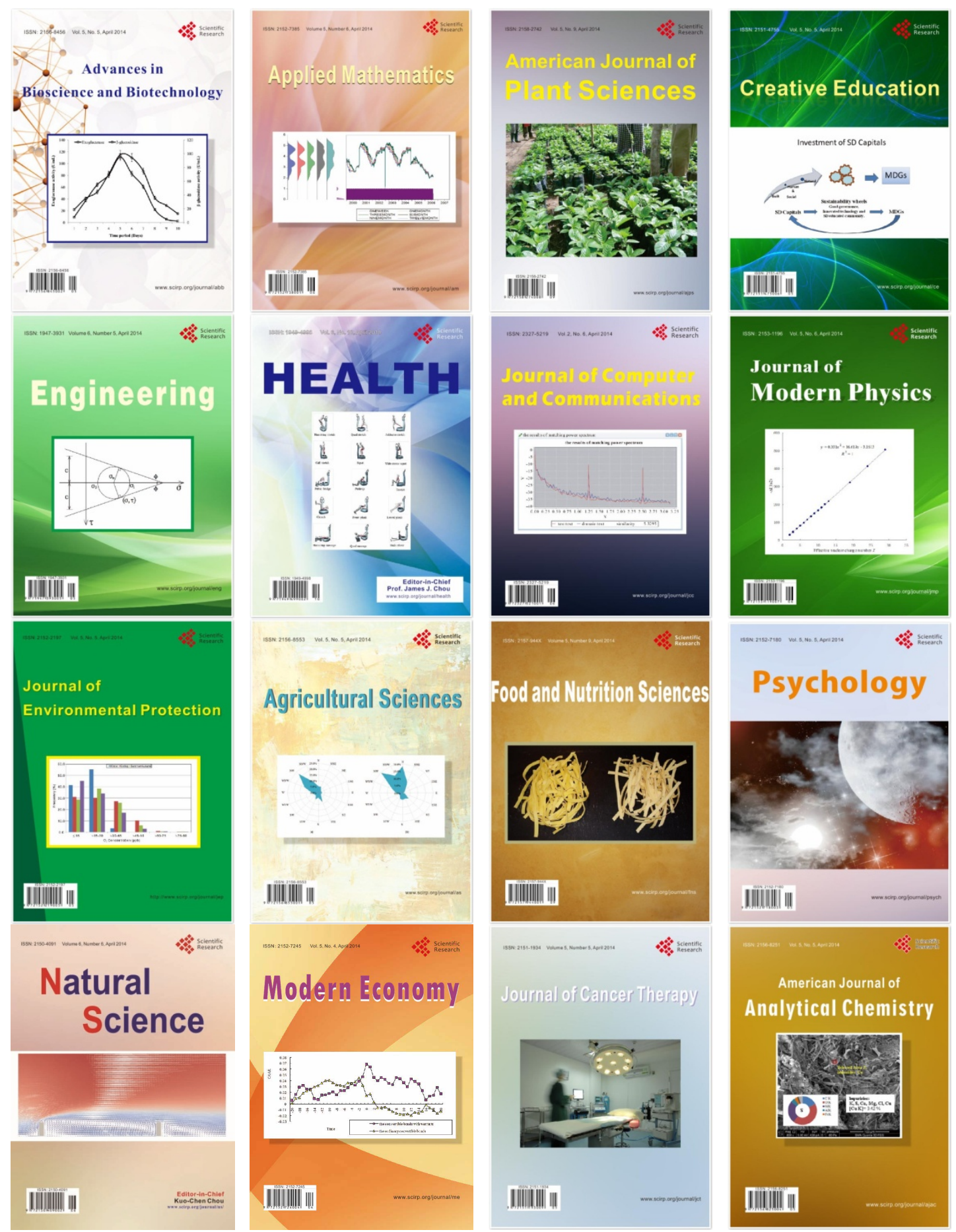